\title{
Violencia de género: reflexiones sobre expresiones verbales presentes en la familia y las instituciones educativas
}

Fecha de recepción : 16 de julio de 2019 • Fecha de aceptación: 16 de octubre de 2019 • Fecha de publicación: 13 de enero de 2020

Adriana Elizabeth Aroca Fárez Universidad Técnica del Norte, Ecuador. aearoca@utn.edu.ec https://orcid.org/0000-0001-6361-3996

\section{Resumen}

Este estudio se ha diseñado bajo un enfoque de análisis documental, de corte transversal, y tiene como objetivo caracterizar las expresiones verbales dentro de la violencia de género presentes en la familia y las instituciones educativas; como instrumento de recolección de datos se ha utilizado la evidencia bibliográfica científica lo más posible actualizada sobre el tema planteado; la investigación aborda la temática desde la familia como núcleo de la sociedad y considerando como lugar donde se origina la violencia de género, para luego develar la presencia del acoso escolar o bullying en las instituciones educativas; todo este análisis transversaliza la situación en materia legal y acciones en pro de buscar soluciones al problema en cuestión. Una de las principales conclusiones a que llega este trabajo es que la violencia verbal causa daño psicológico y está presente en la familia y en la escuela, que por su carácter de no dejar evidencia física en la victima pasa desapercibido por padres y profesores, quienes lo conciben como una normal actitud según las etapas de crecimiento y grupos de edad donde se desarrollen. La investigación abre un camino para nuevas documentaciones científicas que permitan conocer más a fondo el origen de la problemática y que faculten tomar las mejores decisiones para la erradicación o mitigación de la violencia hacia la mujer en la sociedad. 


\begin{abstract}
This study has been designed with a cross-sectional approach to documentary analysis, and aims to characterize the verbal expressions present in the family and educational institutions; as a data collection instrument, the most up-to-date scientific bibliographic evidence on the proposed topic has been used; the research addresses the issue from the family as the core of society and considering as a place where gender violence originates, to later reveal the presence of bullying or bullying in educational institutions; All this analysis cross-cuts the legal situation and actions in favor of finding solutions to the problem in question. One of the main conclusions reached by this work is that verbal violence causes psychological damage and is present in the family and in school, which due to its nature of not leaving physical evidence on the victim goes unnoticed by parents and teachers, who they conceive as a normal attitude according to the stages of growth and age groups where they develop. The research opens a path for new scientific documentation that allows to know more about the problem and that empowers to make the best decisions for the eradication of violence against women.
\end{abstract}

Keywords: verbal expressions, gender violence, family and school. 


\section{Introducción}

El presente trabajo de investigación aborda el tema de violencia de género, que por su complejidad, solo distingue las expresiones verbales dirigidas a la mujer, presentes en la familia y en la escuela.

La violencia verbal a diferencia de la violencia física no deja evidencia de la agresión, sin embargo, su indicios de presencia son reflejados psicológicamente en la víctima, que en el caso de los escolares impacta en deficientes rendimientos y deserción, entre los más principales, que luego se manifiestan en una antipatía social, y de hecho favorecen a un continuismo generacional.

La violencia a pesar de ser un problema de derechos humanos debe darse la debida importancia dentro del ámbito social (educativo) y de salud pública, considerando que en el Ecuador una de cada tres mujeres y uno de cada dos niños o niñas y adolescentes, refieren haber vivido o sufrido algún tipo de violencia intrafamiliar, sin mencionar las experiencias de violencia que se viven en otros ámbitos públicos, privados e institucionales de la sociedad.

Si bien es cierto en el Ecuador a más de la Constitución que garantiza protección a los ciudadanos, existen varias leyes que sancionan la violencia en todas sus formas, y que por otro lado, promulgan el respeto a la integridad personal, como la Ley Orgánica de Educación Intercultural (2011), que contempla como fin de la educación la protección y el apoyo a las y los estudiantes en casos de violencia, cuya erradicación es una obligación así como velar por la integridad psicológica y sexual de los integrantes de las instituciones educativas (artículos 3 literal m y 6 literal h).

La investigación espera contribuir con un breve análisis que permita reflexionar y actuar, tal vez con la motivación hacia nuevos estudios que profundicen el conocimiento de una realidad que a más de ser debatida si es competencia de los roles de género debe ser considerada como una intromisión a la integridad de la mujer; o quizá infundir en el sistema educativo procesos intencionados que si no le es permitido erradicar al menos disminuir los índices de acoso escolar.

En hora buena, que en la actualidad y gracias a varios estudios se pueda determinar que tanto en hogares como escuelas la violencia de género baja cuando los progenitores tienen niveles altos de estudio (Camacho, 2014).

\section{Materiales Y Métodos II}

\subsection{Tipo de investigación}

La investigación es de tipo documental y descriptiva, ya que consistió en una revisión bibliográfica para de esta manera profundizar en el análisis reflexivo a partir de una descripción de las características del objeto de estudio.

\section{$2.2 \quad$ Método}


Se ha utilizado el método analítico sintético, por permitir dar el enfoque deseado en la investigación, es decir revelar los componentes para luego facultar una interpretación de la realidad.

\subsection{Pregunta de investigación}

¿Cómo se caracterizan las expresiones verbales presentes en la familia y las instituciones educativas?

\section{Fundamentos Para El Análisis Y Reflexión III}

\subsection{Identificación de la violencia de género en la familia}

La Constitución de la República del Ecuador (CRE) (2008), reconoce a la familia como un núcleo fundamental de la sociedad, otorgándole responsabilidades dentro del proceso educativo, las cuales promuevan de forma prioritaria el desarrollo integral de las niñas y niños.

En este sentido, la familia representa a la sociedad en todas sus dinámicas sean funcionales o disfuncionales, cuyas acciones procuran encaminar a una coexistencia armónica entre sus miembros.

La familia acentúa más responsabilidades conforme el número de miembros sigue creciendo, es decir, el advenimiento de los hijos (as); en este escenario de encargos a la familia, se concibe a la educación como la más importante entre la protección física, el resguardo de la vivienda, la alimentación, salud y el afecto de padres, porque esta se convierte en el principal canal de comunicación con la sociedad.

Al respecto, Planiol y Ripert (2002), citados en Gómez y Villa (2014), señalan que la familia es:

(...) el medio específico en donde se genera, cuida y desarrolla la vida. En este sentido se convierte en el "nicho ecológico por excelencia, y por qué no, en la primera escuela de la humanización, de transmisión generacional de valores éticos, sociales y culturales que aporta un sentido mucho más amplio a la misma existencia humana".

La educación en la familia, por tanto, es un motor evolutivo que condiciona actitudes y aptitudes, en todo el ciclo de las generaciones.

Ahora bien, comprendiendo a la familia como una integración de hombres y mujeres, sean padres y madres o hijos e hijas, se evidencia la diferenciación de trato y acción en el día a día, y que luego marcan las implicaciones negativas en cuanto al rol y poder de género, que "de los lugares públicos y privados en los que ocurre la violencia de género, donde se produce con mayor intensidad es en la familia" (Loreta y Quiroga, 2013, citado en Nares, Martínez y Colín, 2015).

La violencia de género, distinguida en este estudio como la violencia de género hacia la mujer, puede circunscribirse como todo acto de agresión sea este físico, sexual, verbal, y psicológico, que 
trae consigo secuelas de sumisión, sin dar lugar a una defensión por parte de la agredida, dando paso a una aceptación de normal comportamiento tanto del hombre como de la mujer.

Para aclarar el panorama anterior, es necesario expresar en palabras de Mateo y Espinar (2007) que:

Hablar de género supone distinguirlo del término sexo. Sexo se emplea para hacer referencia a la base biológica de las diferencias entre hombres y mujeres, mientras que género hace referencia a la construcción sociocultural sobre la base biológica, es decir, a los roles, funciones, comportamientos, actitudes, identidades, etc. que las sociedades adjudican a cada sexo y que los seres humanos aprenden e interiorizan.

La violencia contra la mujer en la familia obedece a un proceso histórico, que aparece justamente en la repartimiento de actividades dentro del hogar como fuera de él; por ejemplo, en un estado primitivo quien caza y provee de comida es el hombre, quien cocina es la mujer; la provisión de pieles está dada por el hombre y la confección de vestimenta por la mujer; en igual forma puede distinguirse que mientras los hombres permanecían fuera del hogar sea en tiempos de paz o de guerra, las mujeres se ocupaban del cuidado y educación de los hijos e hijas, mantenimiento de viviendas por más precarias que estas hayan sido, y también en la domesticación de animales y plantas.

En todo este proceso evolutivo de miles de años, es posible que la naturaleza genética condicione el rol de género, que al devenir de los tiempos se enmarcó en una arbitrariedad social, que hoy se lo debate como violaciones de los derechos humanos, que ha decir de Rico (1996), y dentro de los términos que compete al estudio, "la mayoría de las lesiones de los derechos de las mujeres y de las discriminaciones y abusos de los que son objeto se deben específicamente a su condición de mujer".

En realidad somos hombres o mujeres desde que nacemos hasta que morimos, que pasemos por procesos de desarrollo como bebe, niño(a), adolescente, joven adulto y adulto mayor, es otra cosa; por lo anterior se puede inferir que una mujer es vulnerable a sufrir violencia de género en toda época de su vida, es decir, "la violencia sobre las mujeres no es una cuestión biológica, sino de género" (Hernández, Magro, \& Cuéllar, 2015), y que de presentarse en la familia y en etapas de la niñez, se convierte en un problema grave que "afectan negativamente al desarrollo y socialización de los niños y niñas" (Gelles, 1993, citado en Alonso y Castellanos, 2006); además está presente en "todos los niveles educativos y socioeconómicos, en todas las religiones y en todos los grupos étnicos y raciales" (Bernal \& Vaca, 2008).

Entre las evidencias que permiten observar la presencia de expresiones de violencia de género en la familia puede considerarse cuando "Ios niños son con frecuencia testigos de violencia en el seno de las casas y son maltratados a su vez, siendo, por tanto, víctimas que en un futuro pueden repetir conductas violentas" (Rodríguez, 1991).

Uno de los maltratos es sin duda el verbal, el cual puede considerado como la ofensa propinada por el lenguaje vulgar caracterizada por gritos, insultos, órdenes y amenazas que pueden provenir 
de los padres hacia los hijos(as) y entre hermanos, estos considerados como parientes más cercanos que conviven dentro del hogar.

En la violencia es obvio que existe un agredido y un agresor, pero ¿qué motiva al agresor a utilizar expresiones de intimidación?, tal vez ser obedecido e identificarse que es él quien manda, por así decirlo, o buscar una manera que al parecer puede concebirse como menos hiriente para mantener la disciplina.

Por lo expuesto se entendería que la familia es un entorno donde existen normas que se respetan por orden de autoridad y jerarquía, y que de una u otra manera siempre habrá un castigo mayor que pasa del uso verbal al físico o restrictivo, dicho de una manera coloquial.

En este sentido la UNICEF (2014), señala que:

Educar a los hijos para auto-controlarse y comportarse adecuadamente es una parte integral de la disciplina en todas las culturas. Los padres guían al niño para que sepa cómo manejar sus emociones y conflictos de manera que favorezca la sensatez y la responsabilidad, además de la autoestima, dignidad e integridad física y psicológica del niño. Sin embargo, con demasiada frecuencia, los métodos educativos se basan en el uso de la fuerza física o la intimidación verbal. En muchos casos, no es una decisión meditada sino simplemente la consecuencia de la frustración o enfado de los padres o la falta de conocimiento de métodos educativos no violentos.

El escenario expuesto puede ayudar a identificar tres aspectos que envuelven la violencia verbal, así: la desobediencia o indisciplina presente en todo nivel de edad sea hombre o mujer; padres sin educación para ser padres; y la inexistencia de escuelas para padres.

Para el primer aspecto, el no acatar la orden superior es un acto de desobediencia, lo cual conlleva al plano de rebeldía; la humanidad en su contexto histórico tiene innumerables ejemplos de sublevación ante la imposición de la idea ejercida ante todo por el poder del conquistador, el potentado, el dictador, el patrón, el padre, la madre, el hermano(a) mayor, etc. etc. es decir, "la capacidad de desobedecer está en el núcleo de la condición humana" (Ballesteros, 2014).

En tal virtud y llevando el caso al entorno familiar, resulta que el niño(a), debe formarse en un comportamiento de armonía hacia su interior y su inmediato exterior que hasta que no tenga edad escolar será su hogar, luego la escuela y la sociedad en general; la disciplina ejercida en este periodo debe tener límites, que cuando son sobrepasados y especialmente conocidos por la autoridad jurídica son procesados los agresores de manera civil o penalmente; así: el Código Orgánico Integral Penal (2014), establece delitos y contravenciones referidos a la violencia contra la mujer o miembros del núcleo familiar, y el Código Civil (2016), señala que como causa de divorcio a "Los tratos crueles o violencia contra la mujer o miembros del núcleo familiar".

El segundo aspecto, se enmarca en la educación anterior al hecho de ser padre o madre; es posible que la única preparación ante la responsabilidad de la crianza de los descendientes sea la asimilada en sus hogares, que sin duda alguna no habrá hogar con igual formación. "Las funciones 
educativas de los padres no se pueden delegar a otras instituciones. La familia, los padres, educan siempre, aun cuando crean que no lo hacen" (Gallo, 2014).

Lo que muy acertadamente puede esperarse es que la forma y el fondo de la responsabilidad familiar sea muy pertinente a una realidad social, que de acuerdo a los autores Alonso y Castellanos (2006), se produzca en términos de coherencia y empatía, así:

La coherencia. Los padres deben tener y mantener el mismo criterio, que tiene que ser firme, tiene que tener continuidad y permanencia para no crear confusiones en el niño. Corregir desde el principio las conductas agresivas del hijo, no reírlas como si fueran una "gracia" del niño.

La educación para la empatía y transmitir al hijo un afecto y unos valores basados en la comunicación y el respeto mutuos. La educación para la responsabilidad inculcando el binomio esfuerzo-responsabilidad hacia la colectividad. Los padres han de aprender a decir no al hijo, sin crispación, sin violencia, sin temor a que esta negativa provoque en el niño reacciones negativas en el presente o en el futuro. La permisividad no es educativa; inculcar pautas y pequeños hábitos de comportamiento hechos como rutina pone los fundamentos de una vida futura en que el niño sea capaz de asumir sus decisiones.

Sobre el último punto, resulta evidente que como tal no existen las escuelas para padres; hecho que puede ser asumido por la institucionalidad educadora, de allí la importancia de la educación para la sociedad que contemple la participación de la familia; como señala Thomas Gordon citado en Duran, Tébar, Ochando, Martí, Bueno, Pin, Cubel y Genis, (2002), a los "padres se les culpa, pero no se les educa. La propuesta del Programa de Escuela de Padres es, por tanto, aportar unos conocimientos relacionados con el papel de padres que les puedan servir para mejorar su trabajo como tales".

Las expresiones verbales de género, son a la vez maltrato y violencia psicológica, la cual "constituye toda acción u omisión que cause daño, dolor, perturbación emocional, alteración sicológica o disminución de la autoestima de la mujer o el familiar agredido" (Ley Contra la Violencia a la Mujer y la Familia, (1995), citada en Ministerio de Salud Pública del Ecuador (2009)).

Por último, se hace referencia a una de las recomendaciones que en su momento hiciese el Comité para la Eliminación de la Discriminación contra la Mujer (CEDAW) (2017), dirigida a los Estados partes para que apliquen la siguiente medida preventiva:

Adoptar y aplicar medidas legislativas y otras medidas preventivas adecuadas para abordar las causas subyacentes de la violencia por razón de género contra la mujer, en particular las actitudes patriarcales y los estereotipos, la desigualdad en la familia y el incumplimiento o la denegación de los derechos civiles, políticos, económicos, sociales y culturales de la mujer, y promover el empoderamiento, la capacidad de acción y las opiniones de las mujeres.

\subsection{Identificación de la violencia de género en las instituciones educativas}

Tal como se vio anteriormente el niño(a) en edad escolar, lleva consigo un aprendizaje concebido 
en el seno de su hogar, que de una u otra manera lo comparte con su entorno; en este sentido, para el niño(a) que ingresa a un centro educativo es un nuevo mundo por conocer, también con normas y con diferentes actores que posiblemente luego serán los agredidos o los agresores; que de acuerdo con Díaz (2005), "a lo largo de su vida en la escuela, todos los estudiantes parecen haber tenido contacto con la violencia entre iguales, ya sea como víctimas, ya sea como agresores, ya sea como espectadores, siendo esta última la situación más frecuente” (pág. 20).

En la escuela la violencia de género se conmuta al acoso escolar, entendido como el "marco de una relación de desequilibrio de poder entre pares, agresores y víctimas, en la que tienen lugar actos violentos (físicos, psicológicos o virtuales) que, por su carácter intencional, sistemático y repetitivo, se diferencian de otras formas de violencia escolar" (Ministerio de Educación \& Unicef, 2015).

Una de las manifestaciones del acoso escolar es la violencia verbal, dada a través de insultos, críticas, amenazas, "palabras obscenas, apodos, descalificativos, burlas de algún defecto real o imaginado" (Sánchez, 2016), así como también, "difusión de rumores o secretos personales, mensajes amenazantes o insultantes por medios electrónicos" (Ministerio de Educación \& Unicef, 2015); para Calle, Ocampo, Franco y Rivera (2016) es un tipo de violencia psicológica entre estudiantes, que sucede frecuentemente "en los pasillos o cuando se encuentran en las horas de descanso; momento en el cual los estudiantes se encuentran más desprevenidos, permitiendo actuar con mayor libertad y evidenciar este tipo de violencia" (pág. 22); así como también en la fila, baños, en el aula, cuando la profesora o el profesor se vuelven a la pizarra para dar una explicación, mientras se atiende a alguna alumna o alumno, en los cambios de clase, y en el transporte escolar, a través del móvil y de redes sociales (González, 2009).

Pinheiro (2006), citado en Ungei, Unesco y Afagmr (2015), señala que "Es más habitual que los niños perpetren bullying físico, en tanto que las niñas tienen más probabilidades de usar formas de violencia verbal o psicológica" (pág. 2).

La cifras que conmueven una actuación inmediata, señalan que los niños afectados por bullying verbal, como una "forma de violencia habitual en la escuela, muestran que 246 millones de niños y niñas sufren violencia relacionada con la escuela cada año" (Plan International, 2013, citado en Ungei, Unesco y Afagmr, 2015).

Para el caso del Ecuador, de acuerdo con el Ministerio de Educación y Unicef (2015), determinan que:

En el Ecuador, el nivel declarado de incidencia de violencia escolar entre pares (aquellos estudiantes que han recibido al menos una vez un acto de violencia en el último quimestre por parte de otro estudiante) es del 58,8\%. Esto quiere decir que, aproximadamente, 6 de cada 10 estudiantes entre los 11 y 18 años (entre $8 .^{\circ}$ de básica y $3 .^{\circ}$ de bachillerato) han sido víctimas de al menos un acto violento.

Las formas más comunes de violencia escolar entre pares son de carácter verbal y psicológico, sea por insultos y apodos (38,4\%), rumores $(27,8 \%)$ o agresiones por medios electrónicos $(9,7 \%)$, 
que afectan sobre todo al grupo más joven de estudiantes. También se producen formas de violencia más directas, como sustracción de pertenencias $(27,4 \%)$ y golpes $(10,7 \%)$. Esta última es más común entre la población masculina y entre los estudiantes más jóvenes.

El "maltrato psicológico afecta a las facultades mentales (especialmente cognitivas) y a las morales" (O’Hagan, 1995, citado en Gómez, 2006).

La violencia psicológica en niños(as) y adolescentes, a decir del Ministerio de Salud Pública del Ecuador (2009), tiene como factores de riesgo los siguientes:

- Bajo rendimiento escolar.

- Exigencia académicas excesivas.

- Trastornos en el desarrollo (hiperactividad, crisis de llanto, enfermedades, crónicas, retardo mental, etc.)

- Baja autoestima.

- Pobre vínculo afectivo con los padres.

Para Díaz (2005), el observar una deplorable autoestima en los escolares, tiene como antecedentes familiares, donde:

(...) la ausencia de una relación afectiva cálida y segura por parte de los padres, sobre todo de la madre, que manifiesta actitudes negativas o escasa disponibilidad para atender al niño; y fuertes dificultades para enseñar a respetar límites, combinando la permisividad ante conductas antisociales con el frecuente empleo de métodos autoritarios y coercitivos, utilizando en muchos casos el castigo corporal.

La violencia verbal por ser de carácter de ofensa psicológica, también conocida como violencia invisible, "implica siempre conductas dirigidas a causar un daño en la víctima de muy difícil prueba porque, al no tratarse de menoscabos o lesiones físicas, no quedan huellas visibles en la mujer maltratada" (Hernández, Magro, \& Cuéllar, 2015); "en muchas ocasiones no es identificada como agresión sino como manifestaciones propias del carácter del agresor" (Ministerio de Salud Pública del Ecuador, 2009).

De manera general la violencia en la escuela, vista por Navarro y Galindo (2013) es:

(...) fatal y destructiva para todos. Para los violentos, porque creen que tiene poder y que gozan de impunidad ante hechos inmorales y no optan por tener una aceptación en su grupo social. Para las víctimas, porque afecta gravemente su autoestima y el desarrollo de su personalidad. Para el resto de estudiantes, porque viven en un entorno de temor e injusticia donde prima la ley del más fuerte. Para el profesorado, porque dificulta sus procesos de enseñanza y la convivencia. 
Los profesores al igual que los estudiantes se ubican en el plano de agresores, agredidos espectadores, y en muy pocas ocasiones mediadores o conciliadores de paz; recordemos que también fueron niños(as) escolares y adolescentes colegiales, lo cual conlleva a reflexionar que tal circunstancia de violencia de género es tomada como muy normal; o "están más preocupados por los problemas de coexistencia, debido a la presencia o al empleo de armas y drogas y a los conflictos interculturales" (Górriz, 2009, pág. 33).

Un aspecto muy importante que disminuye la presencia de bullying escolar, explicado por Pérez (2016), dice:

Al analizar la relación entre las características de los profesores y la prevalencia de bullying, se concluye que las características observadas de los docentes como una mayor satisfacción al dictar en el plantel educativo, mayor experiencia y un buen nivel de estudios, se asocian con una disminución en la probabilidad de que sus estudiantes sean víctimas de bullying escolar.

\section{Conclusiones}

En la familia la violencia de género se hace identificable en las expresiones verbales que hacen daño psicológico al niño(a), y que son inducidas por quien cree tener el poder, motivadas para conseguir la obediencia y disciplina, el rompimiento de los límites permisibles conducen en el mayor de los casos al castigo físico, generalmente se acusa a la calidad de la educación recibida o no por los padres que determina el nivel de comportamiento circunstancial y dominio de actitudes, en tal sentido, siempre existirá la necesidad de mantener los vínculos indisolubles entre la familia y la institución educativa mediante la aplicación de escuelas para padres.

La violencia de género bajo expresiones verbales que se presenta en las escuelas lleva su homónimo en acoso escolar o bullying, que hacen daño psicológico al niño(a) u adolescente, perpetrado entre estudiantes principalmente mediante el uso de vocabularios de intimidación, que afecta en su mayoría a las mujeres, desarrollado espontáneamente en toda la dinámica que conlleva la razón de estudiar.

El niño(a) que ingresa a la escuela lleva consigo signos y síntomas de haber padecido agresiones verbales, originadas en el núcleo familiar, que por ser de carácter psicológico afectan la autoestima y personalidad, siendo este tipo de agresión perdurable en el tiempo, y que muy probablemente son replicadas en el seno de la escuela de quien se convierte en agresor.

Para el caso del Ecuador, donde no es ajeno a esta realidad, no es suficiente con la información cuantitativa que en su momento ha sido citada en esta investigación, sino que promulga la necesidad de profundizar en estudios que favorezcan la amplitud del conocimiento sobre esta problemática y de allí catapultar el accionar en pro de la erradicación de la violencia de género concebido en todo su ámbito; ante lo cual se debe reconocer que un país con mayor índice de educación el nivel de violencia de género es bajo.

La norma jurídica en el Ecuador está bien argumentada bajo principios y garantías para una correcta educación y protección de sus habitantes, que por otro lado cuenta con acciones

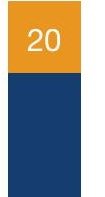


sancionatorias ante la violencia de la mujer, así como también la injerencia institucional para la erradicación de tal problemática presente en las instituciones educativas.

Existe una gran preocupación que trasciende toda frontera política y geográfica para mitigar los daños psicológicos colaterales que ocasiona el maltrato verbal presente en la familia y las escuelas, en este ámbito se han creado instituciones o departamentos de atención internacional como la Unicef, Comité para la Eliminación de la Discriminación contra la Mujer (CEDAW), entre otros, donde a más de reconocer la existencia del problema a través de políticas, hacen recomendaciones para su mitigación o erradicación mundial, en especial para los países poco desarrollados; el inicio de estas ayudas son de manera directa y tienen que ver con la generación de información mediante la aplicación de encuestas nacionales. 


\section{Referencias Bibliográficas}

Alonso, J., \& Castellanos, J. (2006). Por un enfoque integral de la violencia familiar. Psychosocial Intervention, 253-274. Recuperado el 11 de Marzo de 2019, de https://tinyurl.com/y2q9fex7

Ballesteros, G. (2014). Desobediencia civil: un análisis político. Universidad de Granada. Recuperado el 12 de Marzo de 2019, de https://tinyurl.com/y5qcfxtz

Bernal, S., \& Vaca, M. (2008). La familia, víctima de la violencia. Violencia intrafamiliar. Recuperado el 12 de Marzo de 2019, de https://tinyurl.com/yxbbbvoh

Calle, G., Ocampo, D., Franco, E., \& Rivera, L. (2016). Manifestaciones de la violencia escolar en la escuela en perspectiva de los derechos humanos. Un estudio de caso. evista Latinoamericana de Estudios Educativos (Colombia), 13-34. Recuperado el 13 de Marzo de 2019, de https://tinyurl.com/y46I5508

Camacho, G. (2014). La violencia de género contra las mujeres en el Ecuador: análisis de los resultados de la encuesta nacional sobre relaciones familiares y violencia de género contra las mujeres. Recuperado el 1 de Abril de 2019, de https://tinyurl.com/y5ta5u8z

Código Civil. (2016). Ediciones Legales. Recuperado el 12 de Marzo de 2019, de https://asesoriajuridica.utpl. edu.ec/sites/default/files/CODIGO-CIVIL_0.pdf

Código Orgánico Integral Penal. (2014). Registro Oficial №180.

Comité para la Eliminación de la Discriminación contra la Mujer. (2017). Convención sobre la eliminación de todas las formas de discriminación contra la mujer. Recuperado el 23 de Abril de 2019, de https://tinyurl. com/y5qnheum

Constitucion de la Republica del Ecuador. (2008). Quito: Registro oficial 1449.

Díaz, M. (2005). Por qué se produce la violencia escolar y cómo prevenirla. Revista Iberoamericana de Investigación, 17-47. Recuperado el 13 de Marzo de 2019, de https://tinyurl.com/y4trjh9w

Durán, A., Tébar, D., Ochondo, B., Martí, A., Bueno, F., Pin, G., . . genís, R. (2002). Manual didáctico para la escuela de padres. Recuperado el 13 de Marzo de 2019, de https://tinyurl.com/y4dr6qfe

Espinar, E., \& Mateo, M. (2007). Violencia de género: reflexiones conceptuales, derivaciones prácticas. Papers 86, 189-201. Recuperado el 10 de Marzo de 2019, de https://tinyurl.com/y2sxwcal

Gallo, V. (2014). La tarea de ser padres. Asociación Argentina de Orientadores Familiares.

Gómez, E., \& Villa, V. (2014). Hacia un concepto interdisciplinario de la familia en la globalización. Justicia Juris, 
11-20. Recuperado el 3 de Marzo de 2019, de https://tinyurl.com/yxhazhpz

Gómez, M. (2006). Maltrato psicológico. Cuad Med Forense, 103-116. Recuperado el 13 de Marzo de 2019, de https://tinyurl.com/2g6zd2k

González, V. (2009). Guía para el profesorado sobre acoso escolar: detección, identifi cación, intervención y prevención. (u. c. Gobierno de las Canarias Consejeria de educación, Ed.) Recuperado el 13 de Marzo de 2019, de https://tinyurl.com/yykg84h7

Górriz, A. (2009). Roles implicados en el acoso escolar: comprensión de la mente, maquiavelismo y evitación de responsabilidad. Recuperado el 14 de Marzo de 2019, de https://tinyurl.com/y2ra7ly5

Hernández, C., Magro, V., \& Cuéllar, J. (2015). El maltrato psicológico. Causas, consecuencias y criterios jurisprudenciales. El problema probatorio. Aequitas, 27-53. Recuperado el 14 de Marzo de 2019, de https:// tinyurl.com/y6dsu5wn

La Ley Orgánica de Educación Intercultural . (2011). Registro Oficial № 417.

Ministerio de Educación, \& Unicef. (2015). Una mirada en profundidad al acoso escolar en el Ecuador Violencia entre pares en el sistema educativo. Recuperado el 13 de Marzo de 2019, de https://tinyurl.com/y5p49lav

Ministerio de Salud Pública del Ecuador. (2009). Norma y protocolos de atención integral de la violencia de género, intrafamiliar y sexual por ciclos de vida. Recuperado el 13 de Marzo de 2019, de https://tinyurl.com/ y6njwyl3

Nares, J., Martínez, D., \& Colín, R. (2015). Violencia de género en la familia: perspectiva jurídico penal. C I E N C I A e rg o -sum, 116-124. Recuperado el 4 de Marzo de 2019, de https://tinyurl.com/y5adlh9u

Navarro, R., \& Galindo, S. (2013). El docente frente a los actos de bullying. Recuperado el 15 de Marzo de 2019, de https://tinyurl.com/y37seor2

Pérez, J. (2016). ¿Cómo se relacionan las características de los profesores con el bullying escolar? Desarrollo y Sociedad, 183-230. Recuperado el 14 de Marzo de 2019, de https://tinyurl.com/yywmvt4z

Rico, N. (1996). Violencia de género: un problema de derechos humanos. (CEPAL, Ed.) Recuperado el 11 de Marzo de 2019, de https://tinyurl.com/y4qvsyey

Rodríguez, M. (1991). Violencia Familiar. ZERBITZUAN. Recuperado el 12 de Marzo de 2019, de https://tinyurl. com/y5w9oxgj

Sánchez, R. (2016). Las manifestaciones de la violencia escolar y las estrategias de prevención en la escuela secundaria diurna 249 México Tenochtitlan. Ciudad de México. Recuperado el 13 de Marzo de 2019, de http://200.23.113.51/pdf/31971.pdf 
Ungei, Unesco y Afagmr . (2015). La violencia de género relacionada con la escuela impide el logro de la educación de calidad para todos. Recuperado el 13 de Marzo de 2019, de https://tinyurl.com/y4g8vtqn

UNICEF. (2014). Violencia como método disciplinario en la infancia. Recuperado el 12 de Marzo de 2019, de https://tinyurl.com/y6jmtone 